\title{
A Study of Dewetting on (001) Rutile using AFM
}

\author{
J. L. Riesterer, * S. R. Gilliss, ${ }^{*}$ N. Ravishankar,** and C. B. Carter* \\ *Department of Chemical Engineering and Materials Science, University of Minnesota, 151 \\ Amundson Hall, 421 Washington Ave. SE, Minneapolis, MN 55455 \\ **Materials Science Centre, Indian Institute of Science, Bangalore, India
}

Thin $\mathrm{SiO}_{2}$ films on (001) rutile $\mathrm{TiO}_{2}$ surfaces have been observed to dewet and form classic droplet patterns on the surface after high-temperature annealing. [1-2] The proposed driving forces for the formation of these droplet patterns include surface tension, temperature gradients and composition gradients, (including gradients formed from the dissolution and precipitation of the $\mathrm{TiO}_{2}$ substrate into the $\mathrm{SiO}_{2}$ glassy film [2-3]). Additionally, film thicknesses and substrate orientation may also affect the type of patterns formed during dewetting. Liquid phase sintering (LPS) depends on the wetting/dewetting behavior of the glassy-phase additive. [4] Films dewet to minimize the liquid/vapor interface area. [5]

When a substrate coated with a surface film is at a higher temperature than the surrounding atmosphere the Rayleigh instability results. [6] The thickness variations cause the film to break apart into droplets. [5] For thicker films on a planar substrate, dewetting via the Rayleigh instability occurs more slowly. [5]

Plasma-enhanced chemical-vapor deposition (PECVD) was performed with a $\mathrm{SiH}_{4}$ precursor pumped into the chamber at 200 standard $\mathrm{cc} / \mathrm{min}(\mathrm{sccm})$ and $\mathrm{N}_{2} \mathrm{O}$ pumped at $400 \mathrm{sccm}$. Chamber pressure was $900 \mathrm{mTorr}$ and $20 \mathrm{~W}$ of power was used to drive the deposition. The $\mathrm{SiO}_{2}$ films were deposited at $200^{\circ} \mathrm{C}$. The system was purged with $\mathrm{N}_{2}$ before and after deposition. Film thicknesses were approximately 100 and $200 \mathrm{~nm}$. The samples were annealed at $1600^{\circ} \mathrm{C}$, just above the $\mathrm{SiO}_{2} / \mathrm{TiO}_{2}$ eutectic temperature, for $1 \mathrm{~h}$ and allowed to cool in an air atmosphere. Samples were heat treated in high-purity alumina crucibles. The alumina powder served as an impurity getter. Visible light microscopy (VLM-Olympus BH2) and atomic force microscopy (AFM-Digital Instruments equipped with Nanoscope III using $\mathrm{Si}_{3} \mathrm{~N}_{4}$ cantilevers in contact mode) were used to characterize the films.

The films were expected to dewet in a densely branching, cell-like network with a uniform distribution of droplets on the (001) $\mathrm{TiO}_{2}$ surface similar to that investigated by Gilliss, et al. [2] During the anneal of the initial $200 \mathrm{~nm}$ thick films, $\mathrm{SiO}_{2}$ droplets formed on the substrate surfaces. An AFM montage, shown in figure 1, shows a dewetting front with $175 \mathrm{~nm}$ high droplets. The film is retracting and leaving droplets behind instead of rewetting the substrate. By tracing the droplet patterns in figure 1, the front's motion across the substrate surface may be mapped. Dissolution of Ti into the glass is believed to provide the driving force necessary to initiate turbulent flow in the film. $[2,7]$ After the uniform composition change of the entire film, the film's density is also altered, causing the film to contract on the surface. [8]

In thinner PECVD films, $(\sim 100 \mathrm{~nm})$, a different droplet behavior was observed. $\mathrm{SiO}_{2}$ nanodroplets were found to form on top of the rutile surface facets. Tubes of glass form on the facet crests instead of facet valleys, shown in figure 2, due to electrostatic potentials of the oxygen or titanium surface- 
terminated facets and the need to lower the liquid's surface energy, as in the Rayleigh instability. The deposited film is initially uniform. Upon thermal treatment, the film becomes liquid and surface reconstruction of the slightly miscut substrate begins. The newly formed facets cause the film to become non-uniform in thickness, and thus, set up the Rayleigh instability in the film. On cooling, amorphous silica tubes and droplets form on top of the facets.

\section{References}

[1] N. Ravishankar, et al., J. Euro. Ceram. Soc. 23 (2003) 2777

[2] S. R. Gilliss, et al., Mater. Res. Soc. Symp. Proc. 819, Warrendale, PA, (2004), 165

[3] T. Yamaguchi, and K. Kanai, J. Am. Ceram. Soc. 77 (3) (1994) 847

[4] W. D. Kingery, et al., Introduction to Ceramics, $2^{\text {nd }}$ ed. John Wiley \& Sons, New York, 1976

[5] G. Callegari, et al., Langmuir 18 (2002) 4795

[6] Lord Rayleigh, Proc. Lond. Math. Soc. 10 (1878) 4

[7] W. Vogel, Struktur und Kristallisation der Gläser, Pergamon Press, Leipzig, Germany, (1965)

[8] T. Young, Phil. Trans. R. Soc. Lon. 1 (1805) 65

[9] This research was supported by the U.S. Department of Energy through grants DE-FG0292ER45465-A004 and DE-FG02-01ER45883. Special thanks to Tony Whipple from the Nanofabrication Center for depositing the PECVD films.

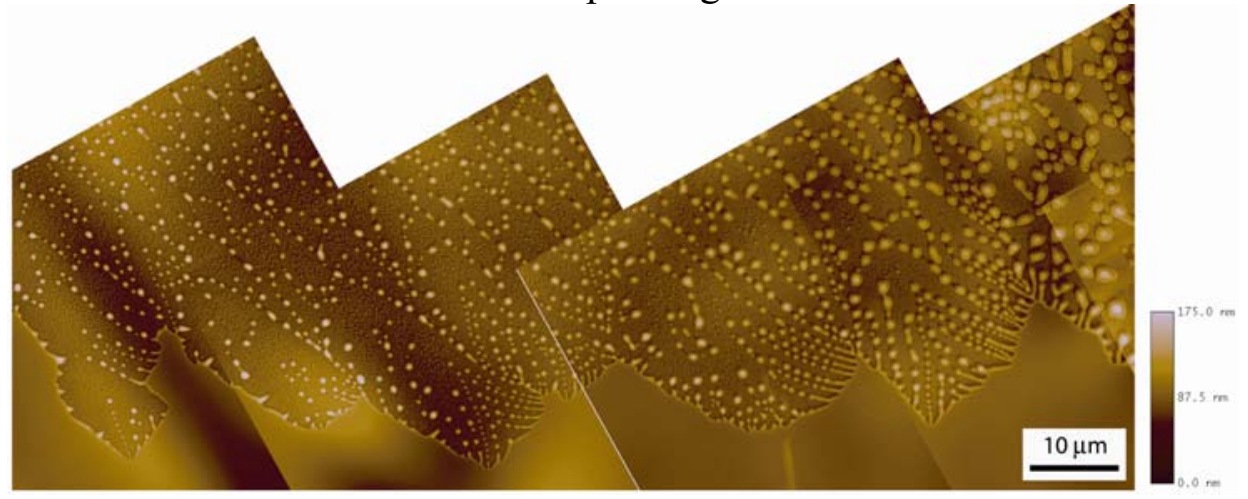

Fig. 1. Contact-mode AFM montage image of a dewetting front in a $200 \mathrm{~nm}$ thick $\mathrm{SiO}_{2}$ film on $(001) \mathrm{TiO}_{2}$. The continuous film has dewet into numerous droplets up to $175 \mathrm{~nm}$ high forming regular patterns.

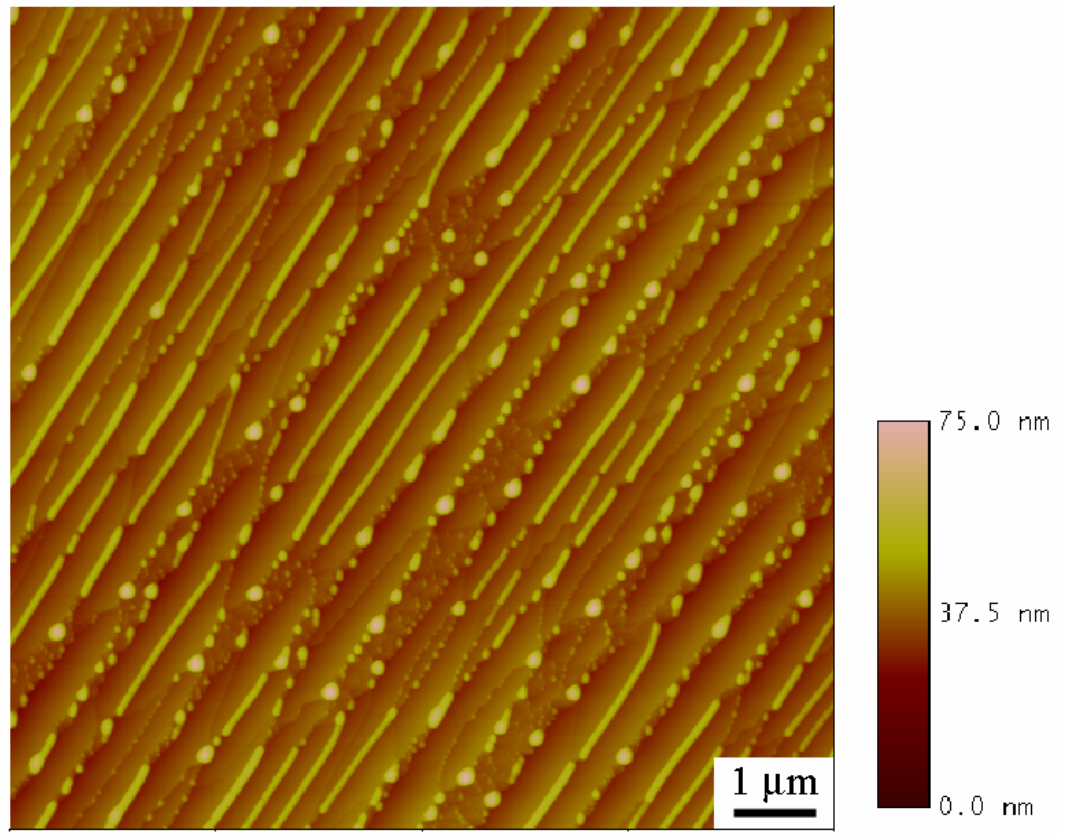

Fig. 2. Droplets are sitting on top of the reconstructed (001) $\mathrm{TiO}_{2}$ surface facet junctions. 